\title{
Perioperative Outcomes Following Surgical Treatment of the Neuromuscular Hip: An Analysis of the National Surgical Quality Improvement Program-Pediatrics
}

\author{
K. Aaron Shaw ${ }^{1}$, Justin M. Hireㄹ, David S. Kauvar², Dana Olszewski ${ }^{3}$, Joshua S. Murphy³ \\ ${ }^{1}$ Department of Orthopaedic Surgery, Dwight D. Eisenhower Army Medical Center, Fort Gordon, GA, USA \\ ${ }^{2}$ Department of Surgery, Uniformed Services University of the Health Sciences, Bethesda, MD, USA \\ ${ }^{3}$ Department of Pediatric Orthopaedic Surgery, Children's Orthopedics of Atlanta, Atlanta, GA, USA \\ Email: Kenneth.a.shaw34.mil@mail.mil
}

How to cite this paper: Shaw, K.A., Hire, J.M., Kauvar, D.S., Olszewski, D. and Murphy, J.S. (2018) Perioperative Outcomes Following Surgical Treatment of the Neuromuscular Hip: An Analysis of the National Surgical Quality Improvement Program-Pediatrics. Open Journal of Orthopedics, 8, 24-32. https://doi.org/10.4236/ojo.2018.81003

Received: November 25, 2017

Accepted: January 19, 2018

Published: January 22, 2018

Copyright (c) 2018 by authors and Scientific Research Publishing Inc. This work is licensed under the Creative Commons Attribution International License (CC BY 4.0).

http://creativecommons.org/licenses/by/4.0/

\begin{abstract}
Background: Surgical treatment for neuromuscular (NM) hip subluxation or dislocation is undertaken to maintain functionality and decrease pain. Longterm complications are well described; however, the acute complication profile is poorly understood. Questions/Purpose: The aim of this study was to identify the rate of and risk factors for complications following surgical treatment of hip subluxation/dislocation, especially as it related to NM children. Methods: Hip reconstruction cases in patients with a NM diagnosis and non-NM patients were obtained from the 2015 American College of Surgeons NSQIP-Pediatric database by CPT code. 30-day postoperative complications were classified according to the Clavien-Dindo system as minor (grade 1 or 2) or major (grade 3+). Patient and surgical factors were assessed in univariate and multivariate logistic regression analyses for association with post-operative complications. Results: 1081 cases were identified (median age 7.7, 55\% female), of whom 420 (39\%) had a NM diagnosis. Overall complication rate was significantly higher in NM patients ( $33 \%$ vs. $19 \%, \mathrm{p}<0.001)$. Numerous factors were associated with postoperative complication on univariate analysis. Multivariate analysis identified NM diagnosis (OR 1.5), age $>6$ years (OR 1.5), or pelvic osteotomy (OR 1.9) as independent risk factors for complication. Conclusion: In pediatric reconstructive hip surgery requiring pelvic osteotomy, a NM diagnosis is associated with an increased risk of 30-day postoperative complications. Older age and increasing surgical complexity were also independently associated with complications. These findings support special attention for the older patients undergoing concomitant pelvic osteo-
\end{abstract}


tomies to minimize complication rate.

\section{Keywords}

Hip Subluxation, Hip Dislocation, Surgical Complications, Pediatric, NSQIP-P

\section{Introduction}

Neuromuscular conditions cause muscle imbalance of the hip which can result in subluxation or dislocation and may decrease quality of life [1]. Pediatric orthopaedic surgeons address these problems with multiple surgical options including soft tissue releases, femoral varus derotational osteotomy (VRDO), pelvic osteotomy, and consideration of open reduction of the hip [2] [3]. Unfortunately, the natural history of neuromuscular hip dislocation is fraught with complications, with reports as high as $81 \%$ of patients developing a post-operative complication [4].

Many studies have evaluated the perioperative complications and risk factors of neuromuscular hip dysplasia corrective procedures at the single institutional level [5]-[16]. Complications can include unplanned readmission, unplanned reoperation, infection, wound complications, pneumonia, nerve injury, implant failure, and blood transfusion. Current knowledge of risk factors for increased complication rate includes presence of tracheostomy or gastrostomy, spica casting, age less than six years, and higher Gross Motor Function Classification System (GMFCS) score, indicating higher severity of disease [16]. However, these risk factors primarily assess the long-term complications as they reflect the risk of recurrent subluxation/dislocation at single institutions.

We sought to perform a retrospective analysis of a prospective nationwide database to assess whether these identified risk factors maintain significance for predicting post-operative complications for pediatric patients undergoing hip reconstructive surgery for neuromuscular hip subluxation and dislocation. We hypothesized that variables reflecting higher GMFCS score (gastrostomy tube, tracheostomy, and ventilator dependence), increasing patient age, and combine femoral and pelvic osteotomies would increase the rate of postoperative complications within 30 days of surgery, in comparison to non-neuromuscular patients.

\section{Materials and Methods}

A retrospective cohort study was conducted using data from the NSQIP-P database. NSQIP-P is a multi-institutional pediatric (age less than 18) surgical outcomes database containing preoperative, surgical, and 30-day outcome variables collected based upon a systematic sampling process to ensure proportional diversity in selection using an 8-day sampling cycle from over 60 pediatric institutions [17]-[22]. NSQIP-P data are continuously audited, validated, and are compiled into Participant User Files (PUFs) for use in quality improvement and sur- 
gical outcomes research. The 2015 NSQIP-P PUF was used for this study. The NSQIP-P PUF data contains no patient identifiers and this research was deemed exempt from institutional review board review by the local Human Subjects Protection Office.

The study cohorts were identified by hip reconstruction procedure current procedural terminology (CPT) codes 27,146, 27,147, 27,151, 27,156, 27,165, 27,258 , and 27,259 . The primary predictor variable of interest was the presence or absence of a neuromuscular diagnosis, which was identified using the NSQIP-P variable of "Cerebral Palsy," Figure 1. Additional variables included age, gender, presence of tracheostomy or gastrostomy tube, seizure disorder, cardiac risk factors, nutritional support, and ventilator dependence (including Bi-PAP or C-PAP use) at the time of surgery. Surgical procedures were characterized as those performed without a pelvic osteotomy (simple-CPT 27,165, $27,258,27,259$ ) and those with a pelvic osteotomy (complex-CPT 27,146, $27,147,27,151,27,156)$. Based upon the results of Ruzbarsky et al. [16], age was stratified into patients 6 years of age and younger or older than 6 years.

The primary outcome was the rate of any surgical complication within 30 days. Postoperative complications were stratified according to the ClavienDindo classification system as modified by Sink for orthopaedic procedures [23], Table 1. Complications were categorized as minor if they were Clavien-Dindo grade two or less, and major for grades three and higher.

Statistical analysis was performed using SPSS statistical package version 24 (SPSS Inc., Chicago, IL). Significance was set at $\mathrm{P}<.05$. Univariate analyses using the Chi-square test were performed to assess for relationships between categorical predictor variables and the occurrence of postoperative complications. Univariates identified as having statistically significant associations with the occurrence of any complication were entered into a stepwise multivariable binary

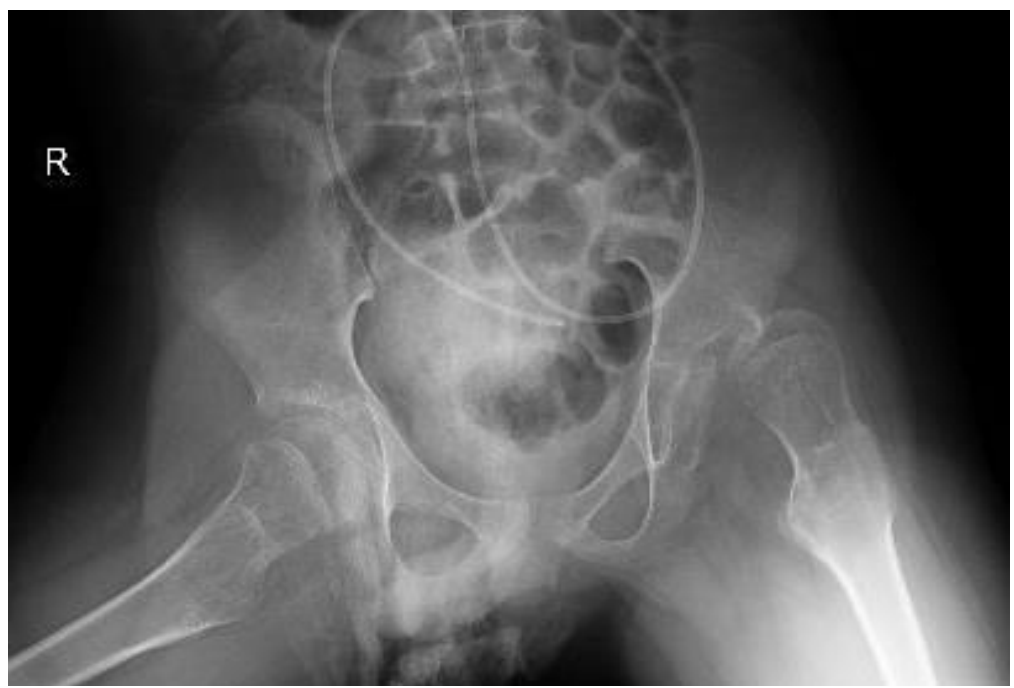

Figure 1. Anteroposterior image of the pelvis in a fourteen-year-old male with cerebral palsy and a Gross Motor Function Classification System level IV who had a painful left hip dislocation. 
Table 1. List of surgical complications as defined using Sink's modification of the ClavienDindo classification system.

\begin{tabular}{cl}
\hline Complication Class & \multicolumn{1}{c}{ Defined Criteria } \\
\hline I & None recorded \\
II & $\begin{array}{l}\text { Superficial infection, urinary tract infection, Seizure, Transfusion, } \\
\text { Venous thromboembolism, Pneumonia }\end{array}$ \\
III & $\begin{array}{l}\text { Wound dehiscence, Neurologic deficit, System sepsis, Reoperation, } \\
\text { Readmission, Pulmonary embolism }\end{array}$ \\
IV & $\begin{array}{l}\text { Reintubation, Coma, Septic shock, Implant failure, Cerebrovascular } \\
\text { accident }\end{array}$ \\
V & Death \\
\hline
\end{tabular}

logistic regression model to determine independent contributions to the occurrence of complications.

\section{Results}

1081 cases were identified (median age 7.7, IQR 4.1 - 11.8 years, 55\% female), of whom 420 (39\%) had a neuromuscular diagnosis. Patients in the neuromuscular cohort were significantly older than the remaining patients (8.99 years, 95\% Confidence Interval 8.65 - 9.33 years versus 7.44 years, 95\% Confidence Interval 7.03 - 7.84; $\mathrm{P}<0.001)$ with a higher percentage of male patients $(60.2 \%$ versus $35.5 \%, \mathrm{P}<0.001)$. Additionally, the neuromuscular patient were more likely to be ventilator dependent $(4.76 \%$ vs. $1.66 \%, \mathrm{P}=0.003)$, have a tracheostomy (3.09\% vs. $4.5 \%, \mathrm{P}<0.001)$, have a seizure disorder $(55 \%$ vs. $7.1 \%, \mathrm{P}<0.001)$, and receive nutritional support $(39.2 \%$ vs. $4.99 \%, \mathrm{P}<0.001)$.

The most common surgical procedure was an isolated femoral osteotomy (42.2\%), followed by a combined femoral and pelvic osteotomy (26.3\%) and pelvic osteotomy (21\%), Table 2. Patients had an average hospital stay of 3.4 days (0 - 85 days) with $98 \%$ of patients discharged home following hospital stay.

Overall, $25 \%$ of patients experienced a complication within 30 days following surgery. The most common complication was a blood transfusion, occurring in $19.7 \%$ of patients. Readmission occurred in 46 patients (4.4\%) at an average of 14.5 days following the primary procedure. The most common stated reasons for readmission were pneumonia (6.5\%), wound complication (6.5\%). However, $39 \%$ of readmissions were for unstated reasons. Unplanned reoperations occurred in 28 patients at an average of 12.4 days following the index procedure. The 2 most common reoperations included reapplication of spica cast (CPT code 29,$325 ; 14 \%$ ) and revision reconstructive procedure (CPT code 27,165; $14 \%)$. When subcategorized according the Clavien-Dindo classification system, there were 232 minor complications (Grade II) occurred in 232 patients and 94 major complications (Grade III and IV) occurred in 36 patients (Table 3).

Complication rates were significantly higher in neuromuscular patients $33 \%$ vs. $19 \%, \mathrm{P}<0.001)$, but the rate of major complications did not differ between 
Table 2. Summary of performed procedures in the patient cohort.

\begin{tabular}{ccc}
\hline Procedure & Frequency & Percent \\
Open Reduction with Femoral Shortening & 38 & 3.5 \\
Isolated Open Reduction & 74 & 6.8 \\
Pelvic Osteotomy & 227 & 21 \\
Combined Pelvic and Femoral Osteotomy & 285 & 26.3 \\
Isolated Femoral Osteotomy & 456 & 42.2 \\
Total & 1081 & 100.0 \\
\hline
\end{tabular}

Table 3. Summary of all complications experienced within the 30-day perioperative period following surgical treatment for hip subluxation or dislocation.

\begin{tabular}{cc}
\hline Complication & Number on Occurrences (Percentage) \\
\hline Blood Transfusion & $213(19.7 \%)$ \\
Unplanned Readmission & $46(4.4 \%)$ \\
Unplanned Reoperation & $28(2.6 \%)$ \\
Pneumonia & $15(1.4 \%)$ \\
Superficial Dehiscence & $14(1.3 \%)$ \\
Urinary Tract Infection & $12(1.1 \%)$ \\
Superficial Infection & $7(0.6 \%)$ \\
Unplanned Re-Intubation & $5(0.5 \%)$ \\
Nerve Injury & $3(0.3 \%)$ \\
Implant Failure & $1(0.1 \%)$ \\
Deep Infection & $0(0 \%)$ \\
Deep Dehiscence & $0(0 \%)$ \\
\hline
\end{tabular}

patient groups $(4.0 \%$ vs. $2.9 \%, \mathrm{P}=0.295)$. Univariate analysis to identify risk factors for surgical complications in all patients identified NM patients, age $\geq 6$, presence of a tracheostomy, gastrostomy, ventilator dependence, need for nutritional support or a concomitant pelvic osteotomy placed patients at a higher risk for any complication, Table 4. Gender and patients with cardiac risk factors did not significantly impact the rate of any post-surgical complications.

The identified risk factors were then entered into a multivariate logistic regression analysis. Multivariate analysis for complication rate identified NM diagnosis (OR 1.5) as an independent risk factor for any postsurgical complications. Additionally, patients aged $>6$ years (OR 1.5), and those undergoing a concomitant pelvic osteotomy (OR 1.9) were independent risk factor in all patients. The remains risk factors (tracheostomy, ventilator dependence, gastrostomy tube, and patients requiring nutritional support) were not predictive of any post-surgical complication.

\section{Discussion}

Surgical management of neuromuscular hip dislocations and subluxation is a 
Table 4. Results of univariate and multivariate analyses for perioperative surgical complications following surgical treatment of hip dislocation or subluxation in pediatric patients. NM, neuromuscular; OR, odds ratio.

\begin{tabular}{ccccc}
\hline & $\begin{array}{c}\text { Complication } \\
(\%)\end{array}$ & $\begin{array}{c}\text { No Complication } \\
(\%)\end{array}$ & P & $\begin{array}{c}\text { Multivariate OR } \\
(\mathbf{9 5 \%} \text { CI })\end{array}$ \\
\hline NM Diagnosis & 33.3 & 19.4 & $<0.0001$ & $1.5(1.1-2.1)$ \\
Female & 22.8 & 27.3 & 0.089 & - \\
Age $>6$ & 28.3 & 18.9 & 0.002 & $1.5(1.1-2.1)$ \\
Pelvic Osteotomy & 31.2 & 19.0 & $<0.0001$ & $1.9(1.4-2.5)$ \\
Tracheostomy & 56.3 & 24.3 & 0.003 & $1.9(0.64-5.6)$ \\
Gastrostomy & 42.9 & 22.8 & $<0.0001$ & $1.5(0.88-2.4)$ \\
Ventilator & 48.4 & 24.1 & $<0.0001$ & $1.9(0.83-4.1)$ \\
Cardiac Risk Factors & 26.5 & 24.6 & 0.68 & - \\
Nutritional Support & 37.4 & 22.0 & $<0.0001$ & $1.3(0.82-1.9)$ \\
\hline
\end{tabular}

challenging endeavor that is plagued with a significant potential for complication as well late resubluxations. We found a $25 \%$ overall rate of complications within 30 days of surgery, significantly higher in the neuromuscular cohort (33\% vs. $19 \%)$. Analysis of risk factors in the patient cohort found that neuromuscular diagnosis, age $>6$ years old, and concomitant pelvic osteotomy were independent predictors for a post-operative complication.

This study is not the first to report a high rate of complications with reconstructive hip surgery for patients with neuromuscular dislocations and subluxations. Numerous authors have reported the complication rates, at an institutional level, for neuromuscular hip reconstructive surgery, ranging from $0 \%-81 \%$ [5]-[16]. Ruzbarsky et al. [16] performed a review of the literature, identifying 16 articles including 851 patients. The cumulative complication rate from their systematic review was $38 \%$, which increased to $54 \%$ when failure to cure was included. Similar to these previous reports, we found that $33 \%$ of pediatric patients with neuromuscular hip dislocation or subluxation in this nationwide patient database developed a post-operative complication within 30 days from surgery.

Complications following reconstructive hip surgery vary based upon the several patient factors. The severity of neuromuscular disease is largely reflected in the patient's ambulatory status and Gross Motor Function Classification System (GMFCS). Nonambulatory patients have been shown to be at significant risk for hip dysplasia, with rate as high as $57 \%$ [16]. At an average of 5.9-year follow-up, Ruzbarsky et al. [16] reported their single center results after hip reconstructive surgery in nonambulatory patients with cerebral palsy. The overall complication rate was $38 \%, 13 \%$ were grade I complications, $18 \%$ of these were grade II, and $2 \%$ were grade III as reported according to the Clavien-Dindo system as modified by Sink [23]. Similarly, we found a 33\% complication rate in our neuromuscular patient cohort, $22.8 \%$ being grade II, $8.6 \%$ grade III.

In addition to the perioperative complications, long-term radiographic out- 
come was reported by Ruzbarsky et al. [16], which when included, raised the overall complication rate to $47.6 \%$. Fourteen patients demonstrated resubluxation at final follow-up with an additional patient developing avascular necrosis. Patients with a tracheostomy and age less than 6 years old were identified as risk factors for resubluxation, characterized as a grade III complication. In contrast, we found that older patients (older than 6 years of age) were independently at a higher risk for experiencing any post-operative complications within 30 days of surgery. However, this discrepancy highlights the different aims of these studies, with age representing the progression of dysplasia exacerbated by muscular imbalance in the setting of continued skeletal growth in Ruzbarsky et al. [16] series whereas our study better characterizes the patient's physiologic reserve within 30 days of surgery with regard to the insult imparted from undergoing a major reconstructive procedure.

There are several limitations in the interpretation of this study. The most pronounced limitation is the mixed population regarding ambulatory status and GMFCS status. Nonambulatory patients and those with higher GMFCS level have been shown to have higher complication rates [24]; however, given the lack of a defined data variable within the NSQIP-P dataset, we were unable to include this in our data analysis. The occurrence of a postoperative complication is restricted to within 30 days of surgery which prevents us from obtaining a true understanding of the long-term complication profile to include resubluxation and the development of avascular necrosis. As a nationwide database, a myriad of surgeons were included with an expected wide variation in surgical technique. Given the coding status, we were unable to sub-define the complication rate by the type of pelvic osteotomy. In addition, surgical blood loss and transfusion protocols were not standardized across participating institutions.

\section{Conclusion}

In conclusion, using a prospectively collected nationwide pediatric database, we defined the overall complication profile in patients undergoing surgical hip reconstruction including the largest patient cohort reported to date. We identified important risk factors that were predictive of short-term postoperative complication within 30 days of surgery, including an underlying neuromuscular diagnosis, patient age greater than 6 years old, and concomitant pelvic osteotomy. Older patients and those with concomitant pelvic osteotomies require particular attention in the post-operative period given their high complication rate. Future studies should focus on approaches to decrease the occurrence of complications in this high-risk group.

\section{References}

[1] Flynn, J.M. and Miller, F. (2002) Management of Hip Disorders in Patients with Cerebral Palsy. Journal of the American Academy of Orthopaedic Surgeons, 10, 198 209. https://doi.org/10.5435/00124635-200205000-00006

[2] Dhawale, A.A., Karatas, A.F., Holmes, L., et al. (2013) Long-Term Outcome of Re- 
construction of the Hip in Young Children with Cerebral Palsy. Bone \& Joint Journal, 95-B, 259-265. https://doi.org/10.1302/0301-620X.95B2.30374

[3] Root, L., Laplaza, F.J., Brourman, S.N., et al. (1995) The Severely Unstable Hip in Cerebral Palsy. Treatment with Open Reduction, Pelvic Osteotomy, and Femoral Osteotomy with Shortening. Journal of Bone \& Joint Surgery, 77, 703-712. https://doi.org/10.2106/00004623-199505000-00006

[4] Mallet, C., Ilharreborde, B., Presedo, A., et al. (2014) One-Stage Hip Reconstruction in Children with Cerebral Palsy: Long-Term Results at Skeletal Maturity. Journal of Children's Orthopaedics, 8, 221-228. https://doi.org/10.1007/s11832-014-0589-9

[5] Al-Ghadir, M., Masquijo, J.J., Guerra, L.A., et al. (2009) Combined Femoral and Pelvic Osteotomies versus Femoral Osteotomy Alone in the Treatment of Hip Dysplasia in Children with Cerebral Palsy. Journal of Pediatric Orthopaedics, 29, 779 783. https://doi.org/10.1097/BPO.0b013e3181b76968

[6] Brunner, R. and Baumann, J.U. (1994) Clinical Benefit of Reconstruction of Dislocated or Subluxated Hip Joints in Patients with Spastic Cerebral Palsy. Journal of Pediatric Orthopaedics, 14, 290-294. https://doi.org/10.1097/01241398-199405000-00003

[7] Canavese, F., Marengo, L. and de Coulon, G. (2017) Results and Complications of Percutaneous Pelvic Osteotomy and Intertrochanteric Varus Shortening Osteotomy in 54 Consecutively Operated GMFCS Level IV and V Cerebral Palsy Patients. European Journal of Orthopaedic Surgery \& Traumatology, 27, 513-519. https://doi.org/10.1007/s00590-017-1902-3

[8] Gordon, J.E., Capelli, A.M., Strecker, W.B., et al. (1996) Pemberton Pelvic Osteotomy and Varus Rotational Osteotomy in the Treatment of Acetabular Dysplasia in Patients Who Have Static Encephalopathy. Journal of Bone \& Joint Surgery, 78, 1863-1871.

[9] Graham, H.K. (2002) Painful Hip Dislocation in Cerebral Palsy. Lancet, 359, 907908. https://doi.org/10.1016/S0140-6736(02)08015-7

[10] Hoffer, M.M., Stein, G.A., Koffman, M., et al. (1985) Femoral Varus-Derotation Osteotomy in Spastic Cerebral Palsy. Journal of Bone \& Joint Surgery, 67, 1229 1235. https://doi.org/10.2106/00004623-198567080-00014

[11] Khalife, R., Ghanem, I., El Hage, S., et al. (2010) Risk of Recurrent Dislocation and Avascular Necrosis after Proximal Femoral Varus Osteotomy in Children with Cerebral Palsy. Journal of Pediatric Orthopaedics B, 19, 32-37. https://doi.org/10.1097/BPB.0b013e3283320c31

[12] Mc Nerney, N.P., Mubarak, S.J. and Wenger, D.R. (2000) One-Stage Correction of the Dysplastic Hip in Cerebral Palsy with the San Diego Acetabuloplasty: Results and Complications in 104 Hips. Journal of Pediatric Orthopaedics, 20, 93-103. https://doi.org/10.1097/01241398-200001000-00020

[13] Miller, F., Girardi, H., Lipton, G., et al. (1997) Reconstruction of the Dysplastic Spastic Hip with Peri-Ilial Pelvic and Femoral Osteotomy Followed by Immediate Mobilization. Journal of Pediatric Orthopaedics, 17, 592-602. https://doi.org/10.1097/01241398-199709000-00005

[14] Noonan, K.J., Walker, T.L., Kayes, K.J., et al. (2001) Varus Derotation Osteotomy for the Treatment of Hip Subluxation and Dislocation in Cerebral Palsy: Statistical Analysis in 73 Hips. Journal of Pediatric Orthopaedics B, 10, 279-286.

[15] Oh, C.W., Presedo, A., Dabney, K.W., et al. (2007) Factors Affecting Femoral Varus Osteotomy in Cerebral Palsy: A Long-Term Result over 10 Years. Journal of Pediatric Orthopaedics B, 16, 23-30. https://doi.org/10.1097/01.bpb.0000228393.70302.ce 
[16] Ruzbarsky, J.J., Beck, N.A., Baldwin, K.D., et al. (2013) Risk Factors and Complications in Hip Reconstruction for Nonambulatory Patients with Cerebral Palsy. Journal of Children's Orthopaedics, 7, 487-500. https://doi.org/10.1007/s11832-013-0536-1

[17] Brighton, B.K. (2015) National Surgical Quality Improvement Program-Pediatric (NSQIP) and the Quality of Surgical Care in Pediatric Orthopaedics. Journal of Pediatric Orthopaedics, 35, S48-S50. https://doi.org/10.1097/BPO.0000000000000548

[18] Dillon, P., Hammermeister, K., Morrato, E., et al. (2008) Developing a NSQIP Module to Measure Outcomes in Children's Surgical Care: Opportunity and Challenge. Seminars in Pediatric Surgery, 17, 131-140. https://doi.org/10.1053/j.sempedsurg.2008.02.009

[19] Minhas, S.V., Chow, I., Feldman, D.S., et al. (2016) A Predictive Risk Index for 30Day Readmissions Following Surgical Treatment of Pediatric Scoliosis. Journal of Pediatric Orthopaedics, 36, 187-192.

https://doi.org/10.1097/BPO.0000000000000423

[20] Molina, C.S., Thakore, R.V., Blumer, A., et al. (2015) Use of the National Surgical Quality Improvement Program in Orthopaedic Surgery. Clinical Orthopaedics and Related Research, 473, 1574-1581. https://doi.org/10.1007/s11999-014-3597-7

[21] Martin, C.T., Pugely, A.J., Gao, Y., et al. (2015) Causes and Risk Factors for 30-Day Unplanned Readmissions after Pediatric Spinal Deformity Surgery. Spine, 40, 238 246. https://doi.org/10.1097/BRS.0000000000000730

[22] Rowell, K.S., Turrentine, F.E., Hutter, M.M., et al. (2007) Use of National Surgical Quality Improvement Program Data as a Catalyst for Quality Improvement. Journal of American College of Surgeons, 204, 1293-1300. https://doi.org/10.1016/j.jamcollsurg.2007.03.024

[23] Sink, E.L., Leunig, M., Zaltz, I., Gilbert, J.C. and Clohisy, J. (2012) Reliability of a Complication Classification System for Orthopaedic Surgery. Clinical Orthopaedics and Related Research, 470, 2220-2226. https://doi.org/10.1007/s11999-012-2343-2

[24] Lonstein, J.E. and Beck, K. (1986) Hip Dislocation and Subluxation in Cerebral Palsy. Journal of Pediatric Orthopedics, 6, 521-526. 